\title{
Editorial on 2020 biomaterials special issue
}

\section{J. Carson Meredith ${ }^{1}$}

Published online: 21 August 2020

(C) Qatar University and Springer Nature Switzerland AG 2020

The word "emergent" refers to that which is undergoing a process of coming into being or becoming more prominent. While the field of biomaterials is well-established, numerous developments are emerging in the field, and the purpose of this special issue is to capture these emergent elements. This special issue presents 10 papers that review advances in biomedical materials with an emphasis on emergent trends in three areas: (i) manufacturing and fabrication, (ii) materials design, (iii) physiological responses to materials. Furthermore, research in this field is becoming more global, with participation from more countries in Asia and the Middle East beyond the traditional contributions of Europe and North America. This volume aims to capture this new global emergence as well, by featuring submissions from diverse places including Belgium, Japan, Malaysia, the Netherlands, Norway, Russia, Singapore, Spain, Turkey, and UAE.

In the realm of manufacturing and fabrication, three papers in this special issue treat emerging aspects of the use of $3 \mathrm{D}$ printing, or additive manufacturing, in production of finely structured biomaterials, such as those useful for tissue engineering constructs. Alagoz and Hasirci review the application of open-source design software and its application to fused deposition modeling as a printing strategy. They detail examples of how interior and exterior architectures are precisely defined to produce implants suitable for specific dimensions and mechanical properties. This review offers valuable insight regarding the authors' perceptions of limitations to the field, which can inform future research. Chief among these, according to the authors, is a lack of variety in biocompatible materials that can also be formed into filaments and melted as required for fused deposition modeling. Gokyurek et al. have contributed a review of the application of $3 \mathrm{D}$ printing to engineered scaffolds for endocrine glands. The paper describes how bioinks containing cells can be used to produce

J. Carson Meredith

carson.meredith@chbe.gatech.edu

1 School of Chemical and Biomolecular Engineering, Georgia Institute of Technology, Atlanta, GA 30332, USA living cell-laden scaffolds in a layer-by-layer procedure, with application to major endocrine glands. Specifically, they present a detailed overview of endocrine function, 3D bioprinting technology, and examples toward printing of vascularized and functional endocrine glands such as the thyroid, parathyroid, and pancreas. Rounding out the fabrication papers, Cristian Felipe-Mendes and colleagues present a review of photopolymerization-based printing processes from conventional stereolithography to two-photon polymerization, capable of producing solid structures with a remarkable control over internal structures. One example reviewed by these authors is two-photon printing of acrylate-modified chitosan that can be blended with poly(ethylene glycol) diacrylate to modulate the biochemical and mechanical properties of the resulting hydrogels. [ref 65 in Felipe-Mendes et al.]

Four additional papers in this special issue treat the subject of biomaterial design via material chemistry and physics concepts. These contributions include the work of Omar et al., who describe an antibacterial and electrically conductive fabric created by coating cotton and polyester with phytic aciddoped polyaniline, which has antibacterial activity against $K$. pneumonia, $S$. aureaus, and E. coli strains. A second paper in this material design and function theme treats the subject of carbonaceous nanomaterials designed for phototherapy, contributed by Betül Gürbüz et al. Photodynamic therapy (PDT) and photothermal therapy (PTT) are two types of phototherapy used to treat tumors by using light to enhance or activate drugs or to generate heat tumors locally. This review examines how combination of PDT and PTT with carbonaceous nanomaterials (CNs) offers additional active complementary and supplementary roles for deep tumors in cancer therapy. For example, CNs including graphene, graphene oxide, carbon nanotubes, and fullerenes can act as efficient photosensitizer carriers for cancer treatment. Hesham Moussa and colleagues discuss the fascinating design of artificial neural network software to control the concentration of drugs released into tumors from liposomes by using ultrasound as an external trigger. This study proposes a feedback drug release controller capable of keeping drug concentration levels, in a tumor site, at or above therapeutic levels. Finally, completing the papers 
in the materials design category, Konashuk and coauthors present an illuminating study on the interrelationship between hierarchical skeletal structure and the local electronic and atomic properties of the bone. To design better biomaterials, for example, bone-mimicking materials, it is required that we also continue to develop imaging and diagnostic methods that reveal more of the microscopic and chemical structure of tissue. By studying the orientation dependence of near-edge Xray absorption fine structure (NEXAFS) of bone tissue in the skeletal frame, they revealed substantial element-specific orientation dependence of the X-ray absorption coefficient. Attributed to emergent X-ray linear dichroism in cortex, this optical phenomenon arises from the relationship between $\mathrm{Ca}-$ $\mathrm{O}$ bonds in the bone and hierarchical organization of the skeleton. An element-specific behavior of the X-ray linear dichroism is detected: the intensity of the $\mathrm{Ca} 2 \mathrm{p}$ is sensitive to orientation of bone specimen while the $\mathrm{O} 1 \mathrm{~s}$ is not. The element specificity provides sensitive probing of (i) chemical bond orientation in the skeleton coordinate frame and (ii) applicability of well-known Wolff paradigm understanding of bone structure at the nanoscale level.

Finally, our special issue is completed by three papers that treat physiological responses and therapeutic issues associated with implants and other biomedical devices. Zeynep Bal and colleagues review bone regeneration in relation to hydroxyapatite-based biomaterials. Hydroxyapatite is the main inorganic component of the bones and has been combined with many natural or synthetic polymers, growth factors, and/or cells to imitate the natural structure of the bone and achieve bone formation and regeneration. A second paper within this physiological response topic is contributed by Shruti Agarwalla et al., which discusses the application of graphene-based materials to reduce the incidence of infection at implant sites. These materials can decrease microorganism viability and biofilm formation, and are poised to emerge as candidates for new preventative and therapeutic strategies to decrease infection-related failure of implanted materials and devices. Finally, Mousumi Sukul and coauthors present a paper that discusses how methacrylation of gelatin hydrogels can improve cell response to implants. Osteoblasts cultured in vitro responded to degree of methacrylation indicating potential tunability of cell response. For example, higher proliferation was observed with higher methacrylation substitution, but cell differentiation behavior was improved for less substitution. It was postulated that the higher proliferation on constructs with a higher percentage of methacrylation is due to the higher stiffness of this gelatin construct, known to positively influence proliferation. 